\title{
Complex multilevel fracture tests of concrete: basic mechanical fracture parameters of standard size specimens
}

\author{
David Lehký ${ }^{1,}$ Hana Šimonová ${ }^{1,{ }^{*},}$ Barbara Kucharczyková ${ }^{1,}$ and Petr Daněk ${ }^{1}$ \\ ${ }^{1}$ Brno University of Technology, Faculty of Civil Engineering, Veveř́ 331/95, 60200 Brno, \\ Czech Republic
}

\begin{abstract}
The aim of the current research is to develop a complex multilevel approach for the experimental-computational determination of mechanical fracture parameters of concrete as a typical quasi-brittle material. This includes testing, advanced evaluation and soft computingbased identification of specimens of multiple sizes in multiple test configurations and analyses of fracture processes using multiscale modelling approaches. The evaluation of a part of an extensive experimental program is presented in this paper. The basic mechanical fracture parameters of the investigated concrete determined on standard test specimens with nominal dimensions of $100 \times 100 \times 400 \mathrm{~mm}$ subjected to the standard three-point bending fracture test are introduced. The results of three different sets of specimens provided with different depths of the initial notch are compared in terms of absolute values of the selected mechanical fracture parameters. The results indicate different sensitivity of particular mechanical fracture parameters in relation to the depth of the initial notch.
\end{abstract}

\section{Introduction}

The theory of concrete fracture, despite all the efforts of numerous researchers, still has failed to provide a clear answer to the problem of fracture processes in concrete. Fracture energy is a fundamental fracture parameter representing cracking resistance and ductility of concrete, and is generally considered as a material property in concrete fracture mechanics and cracking analyses. The most commonly used method for measuring the fracture energy is the work-of-fracture method recommended by RILEM [1]. However, values determined by this method are dependent on the size and shape of the test specimen. Several researchers have analysed this size effect on fracture energy measurements according to the RILEM procedure. They proposed modifications to obtain size-independent specific fracture energy of concrete, see Bažant and Kazemi [2]. Abdalla and Karihaloo [3] showed that the same size-independent specific fracture energy can be obtained by testing only two specimens of the same size but with notches which are well separated.

The size effect is a very important attribute of quasi-brittle (or cohesive softening) fracture mechanics and damage mechanics [4]. It can be explained by two different possible

\footnotetext{
* Corresponding author: simonova.h@ vutbr.cz
} 
approaches - energetic (or deterministic)-based and statistical-based approach, respectively. The deterministic explanation is closely connected with nonlinear fracture behaviour. It represents the transition from ductile failure of relatively small specimens to brittle failure of large structures. The statistical size effect follows the Weibull's assumption of the weakest-link concept [5]. The energetic and statistical aspects are deeply related and it is not possible to separate their consequences at the macro-scale level [6].

When simulating the tensile cracking of concrete, a softening curve represents the relationship between the crack opening displacement and the gradual stress drop after the tensile strength. The crack band model [7] and the cohesive crack model [8] are representative approaches in practical finite element analyses for cracking. It was shown that parameters of softening models including fracture energy can be unambiguously identified neither from the standard size effect tests nor from the tests of the complete load-deflection curve of specimens of one size. A combination of both types of tests is required. Therefore, a comprehensive test program including tests of both types made with one and the same concrete was carried out (Fig. 1).

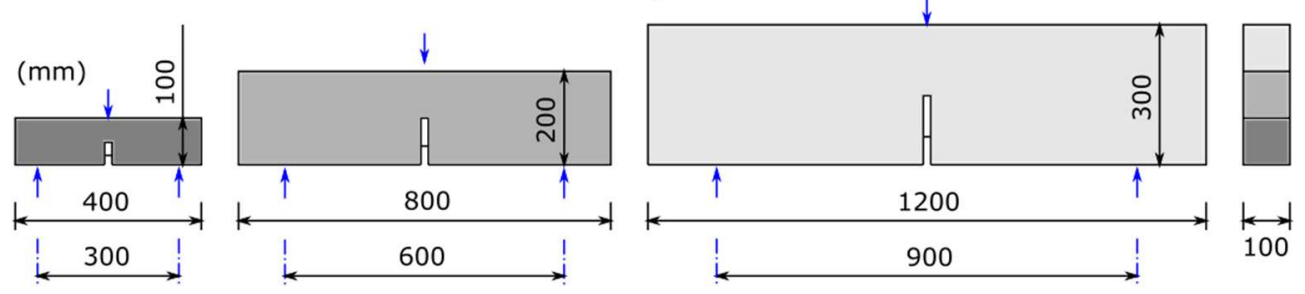

Fig. 1. Specimen of three different sizes each with shallow and deep notch tested in three-point bending configuration.

The aim of the current research is to develop a complex multilevel approach for the experimental-computational determination of mechanical fracture parameters of concrete as a typical quasi-brittle material. This includes testing, advanced evaluation and soft computing-based identification of specimens of multiple sizes in multiple test configurations and analyses of fracture processes using multiscale modelling approaches.

In order to verify the effect of specimen size and geometry on the fracture energy and other mechanical fracture parameters more clearly, three-point bending and wedge splitting tests were performed simultaneously using different specimen sizes and initial notch depths. Regarding the size effect, three different specimen sizes in the ratio 1:3 were tested (see threepoint bending specimens in Fig. 1). In order to follow the procedure of Abdalla and Karihaloo [3], specimens with two well-separated depths of notches were tested for each size and test configuration. The shallow initial notches have a relative notch depth $a / D=0.2$, where $a$ is the notch depth and $D$ is the overall depth of the specimen. The value for the deep notches was 0.5 . In order to expand the knowledge of mechanical and fracture behaviour of the tested concrete, compression tests, splitting tensile tests and non-destructive tests based on the resonance method for the determination of dynamic modulus of elasticity were also carried out in different ages of hardening.

This paper presents the results of one part of the test campaign. On the smallest specimens with a nominal depth of $100 \mathrm{~mm}$ tested in a three-point bending configuration, the effect of notch depth on the basic mechanical fracture characteristics is studied.

\section{Experimental program}

Ordinary plain concrete of $\mathrm{C} 30 / 37$ strength class, which is widely used in engineering practice, was used for the experiment. Before the start of the main experimental part, pilot tests aimed at the design of the appropriate concrete composition were performed. Because 
of the quasi-brittle material, it was necessary to design the concrete composition so as to obtain the whole record of the load $F$ versus displacement $d$ (deflection in the middle of the span length) and load $F$ versus crack mouth opening displacement (CMOD) diagrams including their descending parts during the testing. The maximum grain size of the aggregate was chosen with respect to the dimensions of the test specimens and especially with respect to the dimensions of the ligament above the expected notch. Two concrete compositions with a maximum grain size of 8 and $16 \mathrm{~mm}$ were designed and used for the manufacturing of the test specimens. The aggregate-cement ratio $(a / c)$ was 4.79 and 5.44 (by mass), the binderaggregate ratio $(b / a)$ was 0.51 and 0.46 and the water-cement ratio $(w / c)$ was 0.61 and 0.62 for concrete with the aggregate grain size of 8 and $16 \mathrm{~mm}$, respectively. Plasticizer SikaPlast$501 \mathrm{~W}$ was used in the amount of $1 \%$ of the cement mass to maintain the workability of both concretes. The smallest type of specimens with the dimensions of $100 \times 100 \times 400 \mathrm{~mm}$ was used for the verification of the concrete composition. With respect to the planned experiments, two sets of test specimens featuring two well-separated depths of the initial notches, shallow (S) and deep (L), were manufactured from each concrete and tested under standard three-point bending fracture test (B) at the age of 21 days. The relative depth of the notch was used to distinguish individual sets of specimens. The designations B_100_8_S, B_100_8_L and B_100_16_S, B_100_16_L were used for the specimens with the maximum grain size of 8 and $16 \mathrm{~mm}$, respectively. The selection of the final concrete composition intended for the main experiment was made based on the resulted mechanical fracture characteristics calculated from the recorded $F-d$ and $F-C M O D$ curves obtained from three independent measurements performed for each set of specimens (see sections 3 and 4).

After the final concrete composition was selected, the test specimens were manufactured for the purpose of the main experimental program. In total, 42 specimens with different dimensions intended for the three-point bending fracture test and for the wedge splitting test were manufactured. All these test specimens were tested at the age of about 100 days when the basic mechanical characteristics were already stabilized (to diminish the influence of the age of specimens during testing). This paper introduces a part of the results of an extensive experimental program which focuses on the influence of the depth of the notch on the final values of basic mechanical fracture characteristics. Three sets of test specimens with the dimensions of $100 \times 100 \times 400 \mathrm{~mm}$ with a different depth of the initial notches were subjected to standard three-point bending fracture tests. The reference relative depth of notch 0.33 (middle, "M"), which is widely used by authors for standard fracture tests, was cut along with the above mentioned shallow (0.2) and deep (0.5) notches. The sets of the specimens were labelled as follows: B_100_S, B_100_M, and B_100_L, which indicates the specimens with the depth of $100 \mathrm{~mm}$ with the shallow, middle and deep initial notch subjected to the three-point bending test. Three independent measurements were carried out for each type of the notch.

\section{Determination of basic mechanical fracture parameters}

The mechanical fracture characteristics were determined based on the $F-d$ and $F-C M O D$ diagrams recorded during the three-point bending test of the prismatic specimens with an initial notch. The specimens were subjected to the quasi-static loading using a very stiff multi-purpose mechanical testing machine LabTest 6.250 with the load range of $0-250 \mathrm{kN}$. In all cases, the span length was set to $300 \mathrm{~mm}$ and the loading process was governed by a constant increment of displacement of $0.02 \mathrm{~mm} / \mathrm{min}$ during the whole time of testing. 

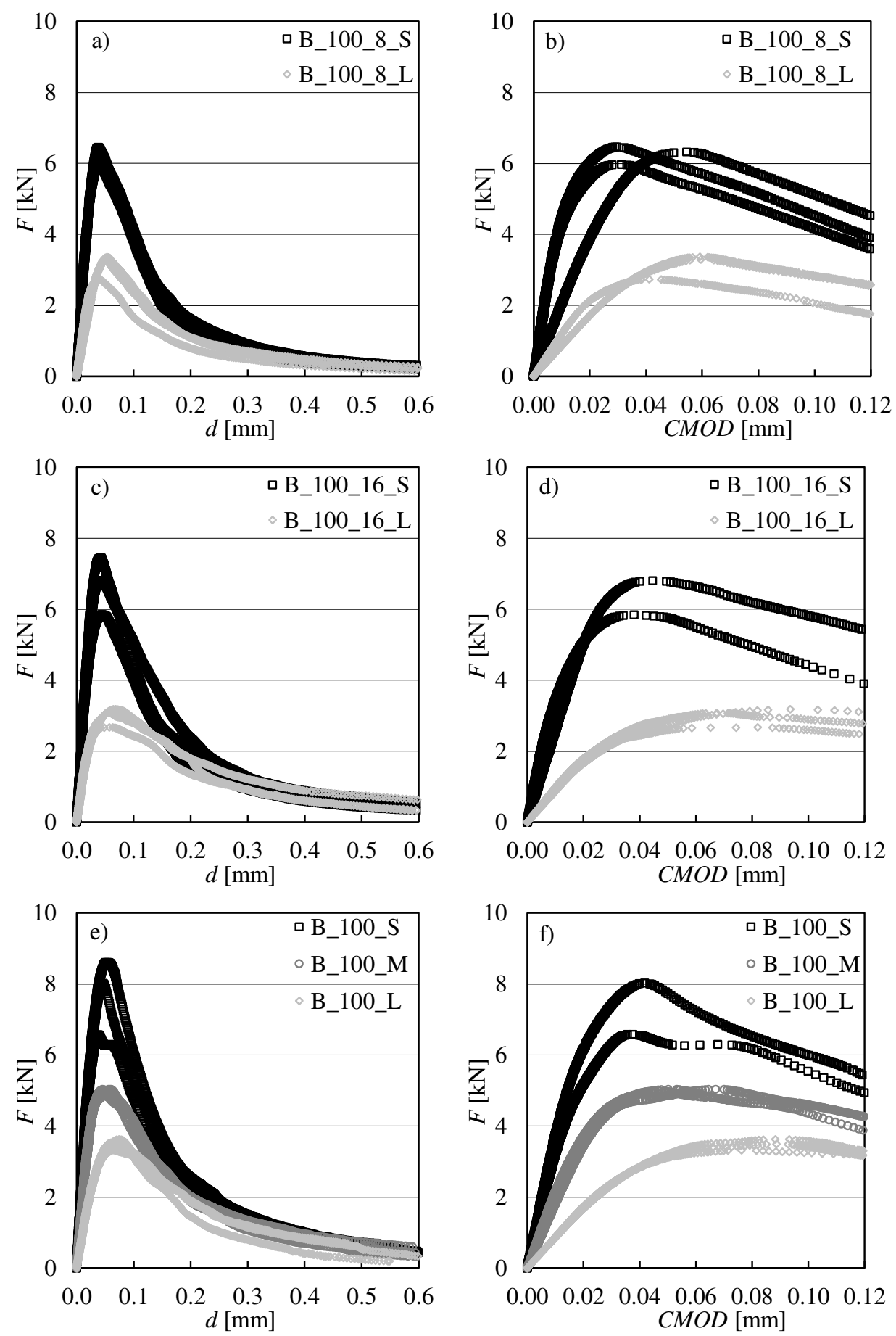

Fig. 2. $F-d$ and $F-C M O D$ diagrams of investigated sets pilot (a-d) and main test (e-f) concrete specimens.

The diagrams recorded during the tests were subsequently processed in order to obtain the appropriate input values for the subsequent evaluation of diagrams using the selected 
fracture model. The processing of diagrams, in this case, incorporated erasing of duplicate points, moving of the origin of the coordinate system, smoothing of the diagram and reduction of the number of points. The processed $F-d$ and $F-C M O D$ diagrams are introduced in Fig. 2. The initial parts of the $F-d$ diagrams were used to estimate the value of the modulus of elasticity according to [9]. The value of effective fracture toughness was determined using the effective crack model which combines the linear elastic fracture mechanics and crack length approaches [9]. The effective crack length, corresponding to the maximum load and related mid-span deflection, had to be calculated to determine the value of fracture toughness. The work of fracture value, corresponding to the area under the $F-d$ diagram, was obtained from the whole $F-d$ diagrams according to the RILEM method [1]. Subsequently, the work of fracture value was divided by the area of a ligament (cross-section of specimens through which the crack grows) and the specific fracture energy value was determined.

The double- $K$ fracture model was employed for the subsequent evaluation of the $F$ $C M O D$ diagrams. The benefit of the double- $K$ fracture model lies in its ability to predict different phases that occur during crack propagation in quasi-brittle material: crack initiation and both stable and unstable crack propagation. The different phases of the fracture process in quasi-brittle material can relate to two parameters: the initiation fracture toughness $K_{\mathrm{Ic}}{ }^{\text {ini }}$ and unstable fracture toughness $K_{\mathrm{Ic}}{ }^{\text {un }}$. The determination of double- $K$ model parameters is based on an approach involving the action of cohesive forces on the faces of the fictitious (effective) crack increment combined with the stress intensity factor criterion (for more details refer to e.g. [10]).

\section{Results}

The average values (determined from 3 independent measurements) and sample standard deviations (depicted as error bars) of selected monitored parameters of concrete specimens with different depths of initial notches determined from $F-d$ and $F-C M O D$ diagrams using the above-described non-linear fracture models are summarized in Figs. 3-7.

Figs. 3 and 4 show the results of the pilot tests aimed at the selection of the concrete composition suitable for wide-range fracture tests. The specimens were tested at the age of 21 days. The sets of concrete were compared in terms of post-peak behaviour and in terms of the absolute values of effective fracture toughness and specific fracture energy. The results show that both concretes exhibited similar post-peak behaviour for both depths of notches (see Fig. 2, a-d). A sudden uncontrolled fall of the descending part of the $F-d$ and $F-C M O D$ diagrams was not observed. The maximum of the recorded load was also similar for both sets of concrete. When comparing the absolute values of the fracture characteristics, it can be stated that the concrete with a maximum aggregate grain size of $16 \mathrm{~mm}$ exhibited a higher absolute value of effective fracture toughness and specific fracture energy for both depths of notches. Based on these results, concrete with a maximum aggregate grain size of $16 \mathrm{~mm}$ was used for the purpose of the main experimental program. 


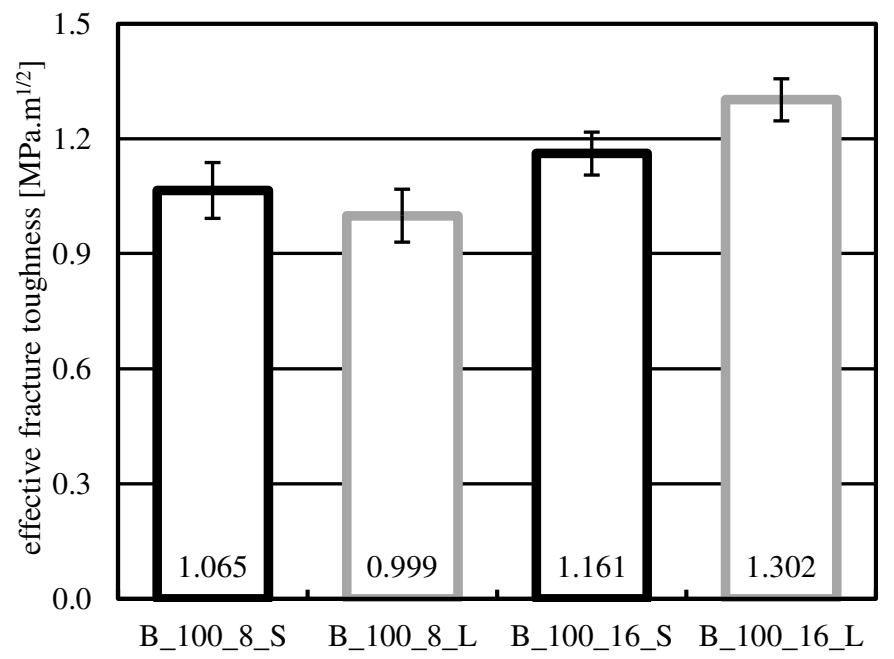

Fig. 3. The effective fracture toughness values of pilot sets of concrete.

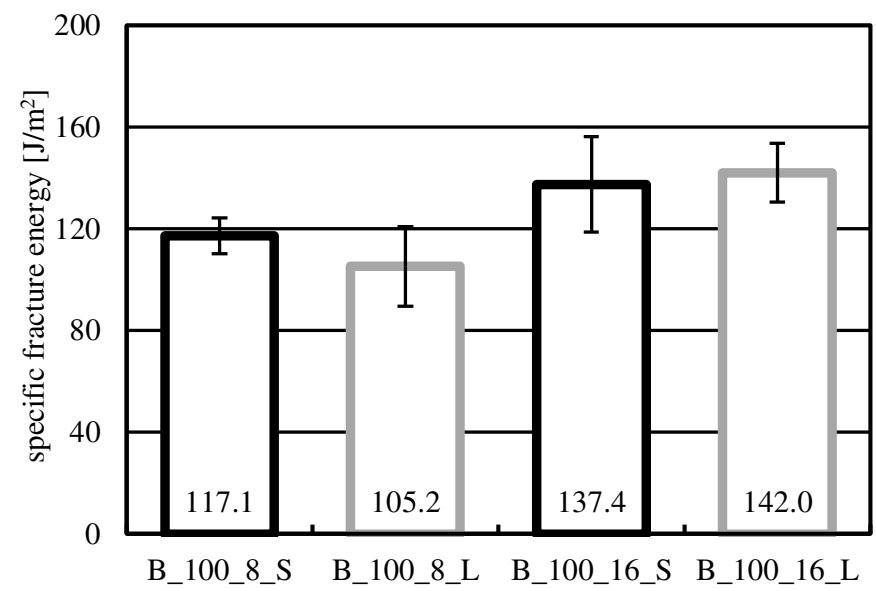

Fig. 4. The specific fracture energy values of pilot sets of concrete.

Figs. 5-7 show the results of the fracture tests performed on the standard test specimens with three different depths of notches at the age of 100 days. The specimens were manufactured from the concrete with a maximum aggregate grain size of $16 \mathrm{~mm}$, the concrete composition corresponds to the composition of the specimens labelled as B_100_16 tested during the pilot tests.

The results show that the value of the modulus of elasticity gradually decreases with the increasing relative depth of the notch (see Fig. 5, a). The modulus of elasticity achieved the highest value of about $34 \mathrm{GPa}$ for specimens with the shallow notch. The value was about $20 \%$ lower in the case of the deep notch. The specific fracture energy values obtained for concrete specimens determined by the work-of-fracture method [1] are presented in Fig. 5, b. The results show the expected decrease in fracture energy with reduced uncracked ligament area, see "M" and "S" specimens. The same trend was not fully confirmed for "L" specimens. Here, the decrease compared to the "S" specimens is relatively small. This can be due to the large variability of the results and also to the relatively small difference between the absolute depths of notches of the smallest specimens. General conclusions will be drawn after analysing the remaining specimen sizes. 

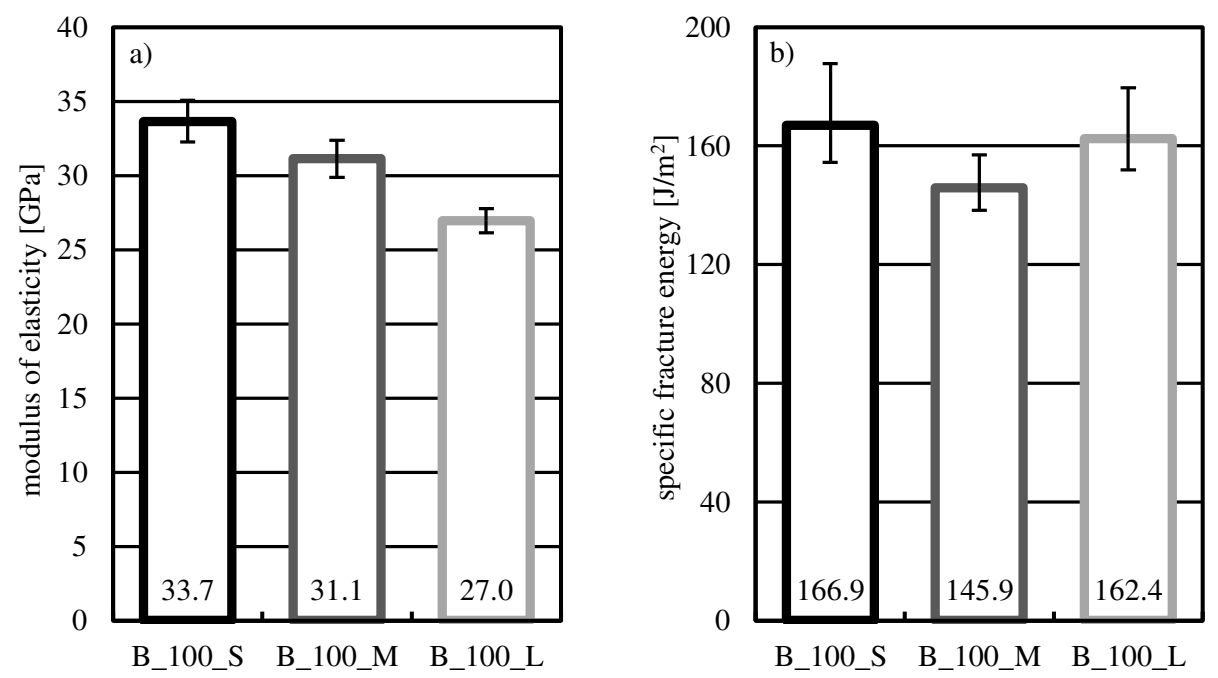

Fig. 5. The modulus of elasticity and specific fracture energy values of the investigated concrete.

The fracture toughness values determined by two different non-linear fracture models are presented in Fig. 6. Based on the results, it can be stated that the values of fracture toughness obtained by both models are similar. The greatest difference between the absolute values of fracture toughness determined on the specimens with different depths of the initial notch is observed for specimens with the shallow and deep notch, and is approx. $20 \%$.
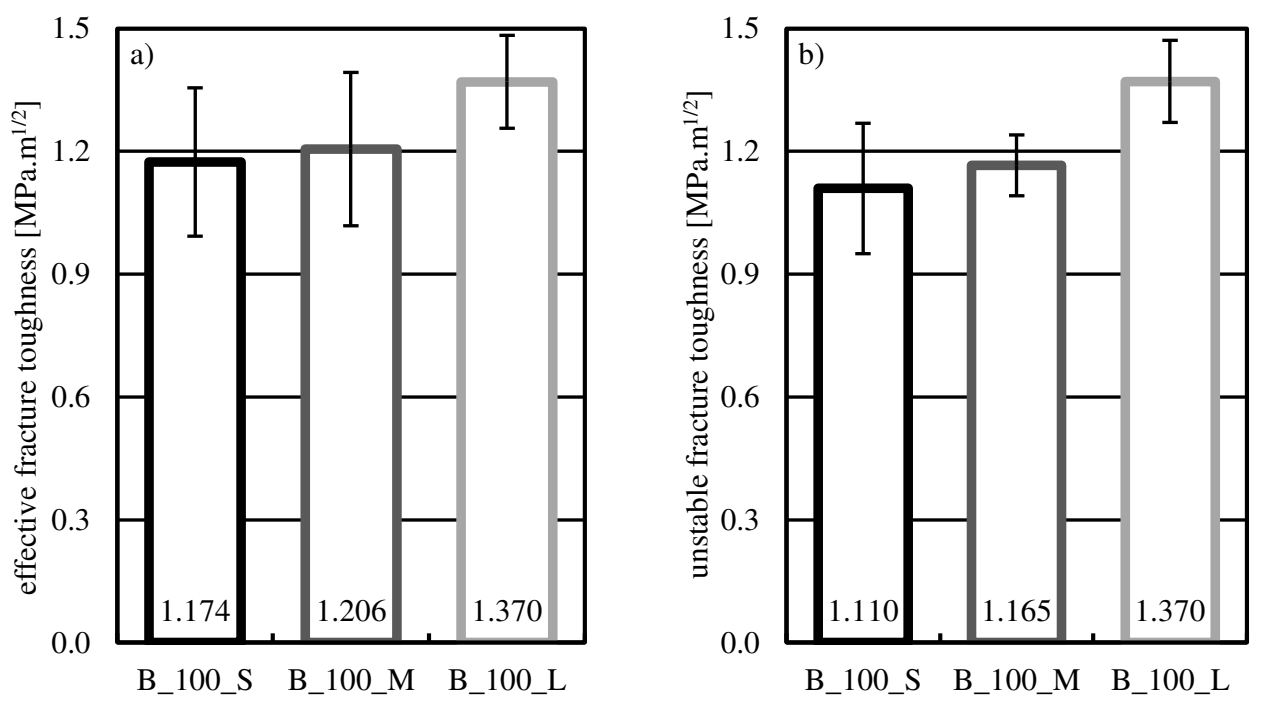

Fig. 6. The fracture toughness values determined by two different fracture models of the investigated concrete.

The $K_{\mathrm{Ic}}{ }^{\mathrm{ini}} / K_{\mathrm{Ic}}{ }^{\text {un }}$ ratio (fracture-toughness ratio, see Fig. 7), which expresses resistance to stable crack propagation, was about $15 \%$ lower in the case of specimens with the deep initial notch than in the case of specimens with the shallow notch. 


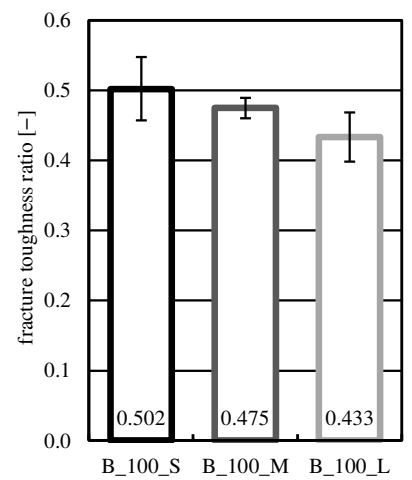

Fig. 7. The fracture-toughness ratio values of the investigated sets of concrete.

\section{Conclusions}

A comprehensive test program was designed and performed as the first step of the development of an advanced experimental-numerical procedure in order to obtain "true" fracture energy and other material parameters independent from testing configuration and specimen size. Such a set of parameters together with an appropriate numerical model can be then utilized for the modelling of real-size concrete structures.

The evaluation of a part of an extensive experimental program is presented in this paper. The basic mechanical fracture parameters of the investigated concrete determined on standard test specimens with the nominal dimensions of $100 \times 100 \times 400 \mathrm{~mm}$ subjected to the standard three-point bending fracture test are introduced. The results of three different sets of specimens provided with different depths of the initial notches are compared in terms of the absolute values of the selected mechanical fracture parameters. Two different non-linear fracture models are used for data evaluation. Based on the results, it can be stated that the values of fracture toughness obtained by both models are similar. The results indicate different sensitivity of particular mechanical fracture parameters to the depth of the initial notch. Because of a relatively high variability of the results, it is essential to evaluate the remaining fracture tests performed on the specimens with different sizes to draw overall conclusions.

This work was supported by the project MUFRAS No. 19-09491S, awarded by the Czech Science Foundation (GACR).

\section{References}

1. RILEM TC-50 FM, Mater. Struct. 18, 107 (1985).

2. Z.P. Bažant, M.T. Kazemi, Int. J. Fract. 51, (1991).

3. H.M. Abdalla, B.L. Karihaloo, Mag. Concr. Res. 55, (2003).

4. Z.P. Bažant, J. Planas, Fracture and size effect in concrete and other quasi-brittle materials (CRC Press, Boca Raton, Florida, and, London, 1998).

5. W. Weibull, Appl. Mech. Rev. 5, 11, (1952).

6. Z.P. Bažant, D. Novák, J. Eng. Mech. 126, 2, (2000).

7. Z.P. Bažant, B.H. Oh, Mater. Struct. 16, 3, (1983).

8. A. Hillerborg, M. Modéer, P.E. Petersson, Cem Concr. Res. 6, 6, (1976).

9. B.L. Karihaloo, Fracture Mechanics and Structural Concrete (Longman Scientific \& Technical, New York, 1995).

10. S. Kumar, S.V. Barai, Concrete Fracture Models and Applications (Springer, Berlin, 